\title{
FOCLAB: A language for computer-controlled psychology research
}

\author{
STEVE MULLEN \\ Laboratory Data Products, Digital Equipment Corporation, Marlboro, Massachusetts 01752
}

\begin{abstract}
The FOCLAB system, a dialect of FOCAL, provides complete real-time experiment control and data acquisition capabilities in the area of experimental psychology. The language provides digital and analog input/output, graphics (including alphanumeries) on a refresh CRT, and a powerful set of time measurement operations. With these facilities FOCAL may be used in cognitive and operant, as well as psychophysiological research. FOCLABB will operate with either PDP-8 or PDP-12 systems, and can control most of the currently existing lab interface options for these computers. The system will operate on $8 \mathrm{~K}$ core-only machines, as well as under $\mathrm{OS} / 8$.
\end{abstract}

Over the last decade, digital computers have assumed an increasingly important function in psychological experimentation. This growth has been brought about by both a trend toward more complex and sophisticated experimental paradigms and a rapid decrease in the cost of computers. During this decade, three minicomputers manufactured by Digital Equipment Corporation, the PDP-8, PDP-12, and PDP-11, have been most employed by psychologists in their research. The FOCLAB system, a dialect of Digital's proprietary FOCAL language, is a software package for the PDP-8 and PDP-12 computers which has been specifically created for psychological research. ${ }^{1}$

The FOCAL language was employed because it is a powerful, yet easy to learn and use, interactive system. A fully interactive language not only allows a new user to rapidly learn to utilize a computer system, but also enables an experienced researcher to continually modify his experiment control programs. The modifications to the $8 \mathrm{~K}$ FOCAL language which constitute the FOCLAB system were made to the LIBRARY command and to the mathematical function routines. The LIBRARY command has been replaced by the LAB statement to which there may be appended (after a space) one of 19 specific commands. These commands and functions are used to control the experimenter's laboratory apparatus via appropriate interface devices. The FOCLAB system provides experiment control and data acquisition facilities for a very wide range of research paradigms.

\section{OUTPUT COMMANDS}

Information may be sent to the experiment via 12 or 24 digital output lines, up to 12 analog signals, as well as

The development of the FOCLAB system was supported by both the University of Minnesota (Human Learning Center) and the Carleton College psychology department (Northfield, Minnesota). a point-plot CRT display. Up to 24 output lines may be turned either on or off by the LAB ACTIVATE and LAB INACTIVATE commands, respectively. Any number of lines may be referenced by a single statement. The LAB CLEAR command will clear all 12 or 24 output lines.

\subsection{LAB CLEAR \\ 1.31 C TURN-ON LINES 1, 5, and 7. \\ 1.33 LAB ACTIVATE 1, 5, 7 \\ 1.35 TYPE "LINES 1, 5, AND 7 HAVE BEEN TURNED ON"! \\ 1.36 LAB INACTIVATE 1,7 \\ 1.39 TYPE "LINES 1 AND 7 HAVE NOW BEEN TURNED OFF!}

Integer values may be directed to one of up to 12 digitalto-analog converters by the LAB LOAD command. The analog voltages may be used, for example, to control, by means of VCOs, the frequencies of audio tones in a perception experiment.

\subsection{ASK "OUTPUT CHANNEL NUMBER $(1-12)$ " N 1.53 ASK "OUTPUT ANALOG VALUE (+ R -2047)" V 1.55 LAB LOAD N, V \\ 1.57 GOTO 1.51}

The LAB LOAD command may be easily altered to allow the 12 or 24 output lines to be simultaneously loaded with the contents of one or two decimal integer (12-bit) values. In this mode, some, or all, of the output lines may be used to control multi-bit devices such as programmable signal attenuators.

Research in human learning and memory processes may make use of a point-plot CRT display to present stimuli and feedback to the subject. There are three FOCLAB statements which provide control over a 
refresh-type CRT. The LAB OUTPUT command will place either a dot or a string of characters on the screen of a CRT at a specified spot, while the LAB TERMINATE and LAB VIEW operations cause the CRT to be blanked or unblanked, respectively. The dot placement requires " $X$ " and " $Y$ " parameters, each with a range between 0 and 1,023 . The text, stored in a temporary buffer, mav be directed to any one of the character lines. A new function, FCRT, allows the user to specify the size of the graphics characters as well as select which of two CRTs will receive the graphics. Depending on the character size, a line may contain 4,8 , or 16 characters. There will be as many character lines as there are characters allowed in each line.

3.71 RESERVE 256 POINTS

SET SCALE $=8$, CRT \#2

3.72 SET D $=$ FCRT $(256,8)$

3.73 SET D $=$ FCRT $(0,2)$

3.74 FOR $\mathrm{K}=0,10,1020$; LAB OUTPUT $0, \mathrm{~K}, \mathrm{~K}$

3.75 C DRAW A DIAGONAL LINE

3.76 LAB VIEW

3.77 TYPE "LOOK AT LINE ON CRT"!

3.78 LAB TERMINATE

3.79 TYPE “LINE NO LONGER ON CRT”!

\section{CONTROL COMMANDS}

To provide rapid access to prestored stimuli, or to temporarily save data, the LAB HOLD statement will insert an integer value into one of the 2,048 locations within a temporary buffer area. A new function, FGET, will retrieve any value which has been stored in this buffer. Such integer values may be $X$ and $Y$ values for display points, or the decimal equivalents of ASCII characters.

5.11 LAB HOLD 1,196

5.12 LAB HOLD 2,197

5.13 LAB HOLD 3,195

5.14 SET D = FCRT $(64,4)$

5.15 SET D = FCRT $(0,1)$

5.16 LAB OUTPUT $3,1,2$

5.17 LAB VIEW

5.18 TYPE 'THE LETTERS 'DEC' ARE SHOWN

IN ROW 2, CRT 1"!

5.19 LAB TERMINATE

A FOCLAB program may be "halted" for up to $2,047 \mathrm{msec}$ by use of the LAB DELAY statement. This command is particularly useful in creating brief output pulses on one or more of the output lines.

7.51 C TURN ON LINE 12 FOR ONE-HALF SECOND

7.53 LAB ACTIVATE 12

7.55 LAB DELAY 500

7.57 LAB INACTIVATE 12
Most operant research is carried out upon several subjects simultaneously. While FOCLAB is not able to support the extensive multi-station control provided by the more specialized state-change languages such as SCAT (Polsen, 1973), SKED (Snapper, 1973), and ACT (Millenson, 1971), it is able to service one to four operant stations. Each station may be under the control of a different schedule programmed in FOCLAB. All station programs must, however, be loaded at the same time. Once loaded, these programs operate under the control of a "time-share" mode provided by the FOCLAB system. A pair of commands, LAB BEGIN and LAB QUIT, initiate or cancel this special time-sharing mode of operation. When the system is in the time-share mode, up to four unique user programs, written with only a few minor restrictions in the FOCLAB language, can be executed "simultaneously."

In addition to the commands which provide control over laboratory apparatus, there are commands which enable or disable the Teletype "echo," stop or start the Teletype paper-tape reader (OMNIBUS processors only), as well as switch input and output operations to highspeed paper-tape equipment. These are the LAB ECHO, LAB NONECHO, LAB WAIT, LAB READ, LAB FAST, and the LAB SLOW commands, respectively. A single ASCII character may be output (to a low-or high-speed device) by using the LAB PUNCH statement.

\section{INPUT FUNCTIONS}

Many of the standard FOCAL language functions have been deleted to allow the addition of new functions. Only the FITR, FSQT, FSGN, and FABS functions remain. The FRAN function has been modified to return a statistically useful random number which is particularly valuable in experimental procedures. Information may be obtained from the subject via 12 or 24 digital input lines, the console terminal keyboard, or up to 16 analog channels.

The status of the 12 primary input lines may be monitored by two different functions. The FAIN function (with a zero argument) returns the number of any line which is or has been active. When the argument is nonzero, the status of all 12 primary input lines is returned as a 12-bit integer.

\subsection{SET LN $=$ FAIN() \\ 6.33 IF (LN) 6.31, 6.37,6.35 \\ 6.35 TYPE! "LINE" LN, "IS, OR HAS BEEN, ON"! ,GOTO 6.31 \\ 6.37 TYPE! “THERE ARE NO LINES”!;QUIT}

The FBIN function is a Boolean function which returns a value of zero (True) if the primary line specified by the argument is, or has been active; or else it returns a minus one (False). This function may be conveniently utilized within an IF statement. 


\subsection{ASK! 'TEST LINE NUMBER $(1-12)$ "' LN \\ $8.33 \mathrm{IF}$ (FBIN(LN)) 8.35, 8.37, 8.37 \\ 8.35 TYPE! "THE LINE IS NOT ON" ;GOTO 8.31 \\ 8.37 TYPE! "THE LINE IS ON” ;GOTO 8.31}

The new FSWR and FADC functions return, respectively, integer values from the front panel switch register or from one of 16 analog-to-digital converters. The switch register provides a simple way of making parameter changes to multiple programs operating in the timeshare mode. Low sampling rate, multi-channel analog input may be used (with the appropriate high-gain amplifiers, filters, and integrators) for biofeedback studies. A single character may be input (from either a low- or high-speed device) by using the FINP function. The FINP function will also return data, which may be a character from a remote parallel-output keyboard, from the secondary 12-bit input device.

\section{TIMING FUNCTIONS}

Because most experiments require many accurately timed intervals, two different functions are provided which $r \in$ ference an interrupt-driven software clock. The FSEC function returns the current value of the "seconds" clock, whereas the FMS function (with a zero argument) returns the value of the "milliseconds" clock. These functions may be used to measure the duration between events, or to provide a specified time interval. The LAB ZERO command resets both clocks to zero and, in addition, initiates a response latency period of up to $4 \mathrm{sec}$. If during this latency period, any one of the 12 primary input lines should become active, or a character should be typed on the console keyboard device, or a bit should become nonzero from the secondary 12-bit input device, the current value of the millisecond clock will be preserved. This value may then be retrieved by using the FMS function with a nonzero argument.

\subsection{TYPE! “WHEN A LETTER IS TYPED PRESS THE SPACE-BAR" \\ 3.71 TYPE “AS FAST AS YOU CAN"!!!!! \\ 3.72 SET ST $=$ FSEC ()$+2.5+5 *$ FRAN () \\ 3.73 IF (FSEC)-ST) 3.73; LAB PUNCH 216; LAB ZERO \\ 3.74 IF (FINP(1)) 3.75, 3.74, 3.75 \\ 3.75 SET RT = FMS (1); TYPE \# "H"!!! \\ 3.76 IF (RT) 3.77, 3.78, 3.79 \\ 3.77 TYPE!! "SORRY, YOU WERE TOO SLOW”! ;GOTO 3.70 \\ 3.78 TYPE!! "DID YOU WAIT TO SEE THE LETTER?”!;GOTO 3.70 \\ 3.79 TYPE!! "YOUR REACTION TIME WAS" RT, "MSEC"!,GOTO 3.70}

The capacity to generate accurately timed stimuli events, as well as measure response latency, allows

FOCLAB to be used in any type of reaction time study. These timings may be made with a resolution of $1 \mathrm{msec}$ if the clock interrupt rate is $1,000 \mathrm{~Hz}$.

\section{EXPANSION CAPABILITIES}

The FX function calls a user-written subroutine (256 words maximum), thus providing the capability to control a special laboratory device or to utilize a peripheral such as a lineprinter, plotter, video terminal, or magnetic-tape drive. The function may also be used to provide a particular service such as signal averaging, or histograms. The latter two operations may make use of the Schmitt triggers provided by the DK8-ES programmable clock option. These triggers are not utilized by the standard version of FOCLAB.

\section{CONFIGURATION GUIDE}

The standard DECUS release of FOCLAB (Version 5.0) has been created for any LAB-8/E system with $8 \mathrm{~K}$ of memory. The specific hardware supported by Version 5.0 is indicated in Table 1 by an asterisk. Naturally any 8K PDP-8 (except the 8/S) which includes these hardware options may use the standard version. If the con-

Table 1

FOCLAB Interface Guide

Interface Devices Release

Clock Options

DK8-ES with the H945 (1,000-Hz clock)

DK 8-EP $(1,000-\mathrm{Hz}$ clock $)$

DK8-EC (500-Hz clock)

DKC8-AA (100-Hz clock)

$\mathrm{KW} 12-\mathrm{A}(1,000-\mathrm{Hz}$ clock $)$

Analog Input Options

AD8-EA + AM8-EA (one/two) + AM8-EC with the H945 Laboratory Mounting Panel

AD8-ES + AM8-EA (one/two) + with the H945

AD8-ES with the H945

AD8-EA + AM8-EA (one/two)

AD8-EA

AD8-A (16 channels) w/wo an H322 + an H323-B

AD01 (with up to 32 channeis)

AD12 (16 or 32 channels)

Digital Input/Output Options

DR8-EC (one/two) with the H945

DR8-EA (one/two) w/wo an H322 each

DKC8-AA w/wo an $\mathrm{H} 322$

DKC8-AA (w/wo an H322) + DR8-EC with the H945

DKC8-AA + DR8-EA (each w/wo an H322)

PDP-1 2 relay register (six SPDT relays)

*@

$*(a)$

\#

CRT Options

VC8-E w/wo (one/two) VR14 displays

VRI2 (one/two displays)

\section{Analog Output Options}

AA50-AP with up to 12 DAC channels VC8-E used as $2 \mathrm{chn}$. DAC (for an X-Y recorder)

AA05 with up to 64 DAC channels

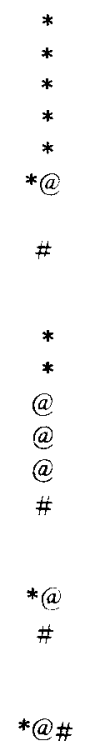

* $L A B-8 / E$ Version @LAB $-8 / A$ Version 
figuration includes any of the other interface options listed in Table 1, the user must write a short patch to overlay the relevant portion of the FOCLAB system. The FOCLAB manual provides the necessary instructions for accomplishing this task. ${ }^{2}$

The FOCLAB system is available from the DECUS library on three media: paper tape, DECtape, ard Floppy disk. The paper-tape release provides only a ready-to-run binary version of the system, whereas the DECtape and Floppy releases also contain the FOCLAB source files. Also included with the DECtape release are the overlay patches necessary to enable FOCLAB to operate on a PDP-12. The PDP-12 options supported by the modified version are indicated by a pound sign. The user may write routines, called via the FX function, to control a LINCtape drive.

The Floppy disk release provides a special version oriented for the LAB-8/A. This version allows the 12-bit digital I/O provided by the DKC8-AA option to be utilized for the primary input and output lines. These lines can be accessed via an H322 screw-terminal panel connected, by a pair of BC08R cables, to the DKC8-AA. The secondary digital device may be a DR8-EC option or a DR8-EA with the BC08R and H322 combination. A $100-\mathrm{Hz}$ crystal clock is also included on the DKC $8-\mathrm{AA}$, but it is not used in the LAB-8/A system. Those options supported by the special LAB-8/A version are indicated by a " $(a$ " sign in Table 1 .

A very inexpensive lab system may be created from an 8K PDP-8/A, an ASR-33 Teletype, and a DKC8-AA option used both as a $100-\mathrm{Hz}$ crystal clock (with a tenfold reduction in time resolution) and as the primary (and only) digital $\mathrm{I} / \mathrm{O}$ device. Patches are included on the Floppy release of FOCLAB to allow the operation of such a minimal configuration.
Although FOCLAB is designed for a system which provides only paper-tape $\mathrm{I} / \mathrm{O}$ (the high-speed option is recommended), the system also operates under OS $/ 8$. The OS/8 system allows both FOCLAB and user programs to be loaded from mass storage files. It is not possible, however, for FOCLAB programs to read and write mass storage files.

The FOCLAB system has been used for 5 years, at several different installations, for research, teaching, and industrial applications. The DECUS library order number for FOCLAB is FOCAL 8-319. A self-teaching manual, also available from DECUS, is designed to allow a new user to learn both FOCAL and the FOCLAB commands and functions.

\section{REFERENCES}

Millenson. J. R. A programming language for on-line control of psychological experiments. Behavioral Science. 1971. 16. 248-256.

Polson. P. G. SCAT: Design criteria and software. Behavior Research Methods \& Instrumentution, 1973, 5, 241-244.

SNAPPER. A. G. Use of a notational system for digital control and recording. Bchavior Re'search Methods \& Instrumentation, 1973. 5. 124-129.

\section{NOTES}

1. For PDP.11 users, the FOCAL, RT-11 system (QJ922) from Digital Equipment Corporation has been specitically oriented for psychological and physiological experiment control and data acquisition. The language features event timing resolutions of $1 \mathrm{msec}$, as well as an alterable character generator for pointplot refresh CRT displays.

2. User-constructed interfaces may be supported by creating the necessary overlay patches. The manual provides the requisite information to accomplish this task. 